\title{
Postdischarge pain, nausea and patient satisfaction after diagnostic and breast-conserving ambulatory surgery for breast cancer: A cross-sectional study
}

\author{
Mi Stjernberg ${ }^{1,2}$ (๑) | Ellen Schlichting ${ }^{3}$ | Tone Rustoen ${ }^{1,2}$ | Berit T. Valeberg ${ }^{4}$ | \\ Milada C. Småstuen $^{1,4}$ | Johan C. Raeder ${ }^{2,5}$
}

${ }^{1}$ Division of Emergencies and Critical Care, Department of Research and Development, Oslo University Hospital, Oslo, Norway

${ }^{2}$ Faculty of Medicine, University of Oslo, Oslo, Norway

${ }^{3}$ Department of Breast and Endocrine Surgery, Oslo University Hospital, Oslo, Norway

${ }^{4}$ Department of Nursing and Health Promotion, Faculty of Health Sciences, Oslo Metropolitan University, Oslo, Norway

${ }^{5}$ Division of Emergencies and Critical Care, Department of Anaesthesiology, Oslo University Hospital, Oslo, Norway

Correspondence

Mi Stjernberg, Division of Emergencies and Critical Care, Department of Research and Development, Oslo University Hospital, v/ Mi Stjernberg, Postboks 4950 Nydalen, 0424 Oslo, Norway.

Email: uxmier@ous-hf.no

Funding information

The PhD-student received a funding from Raagholtstiftelsen when writing the study protocol.

\begin{abstract}
Background: The aims of this study were to assess first day postdischarge pain, nausea and patient satisfaction in ambulatory breast cancer surgical patients, after diagnostic and breast conserving procedures.

Methods: A total of 781 women, aged $18-85$ years were included in this prospective, cross-sectional study. All patients received standardized multimodal pain prophylaxis with paracetamol, COX-II inhibitor, dexamethasone and wound infiltration with local anaesthetics. Nausea prophylaxis was provided with ondansetron. Most patients received general anaesthesia with propofol and remifentanil. Data were collected using a validated questionnaire during telephone follow-up on the first postoperative day.

Results: The response rate was $94.5 \%$. NRS $\geq 4$ was reported by $5.3 \%$ at rest, by $17 \%$ during activity and by $30.7 \%$ as the worst pain score. Young age was strongly associated with more pain both at rest, during activity and regarding worst pain since discharge. Postdischarge nausea was present in $17.8 \%$, and vomiting in $1.2 \%$. High pain score during activity and higher level of worst pain, were associated with nausea. There was no association between nausea and age, type of anaesthesia, surgical procedure or pain at rest. Patient satisfaction was high (97.8\%-99.7\%) regarding information, time for discharge and overall satisfaction. Conclusion: Pain scores and incidence of nausea were generally low on the day after surgery. Young age was a strong predictor for postdischarge pain. A high worst pain score and high pain score during the activity were associated with postdischarge nausea. Patient satisfaction was high.
\end{abstract}

\section{KEYWORDS}

ambulatory surgery, breast cancer, cross sectional study, patient satisfaction, postdischarge nausea, postdischarge pain, postoperative symptoms, telephone follow-up

\section{Editorial Comment}

This prospective study shows that in this single centre sample population, pain and nausea are rare and overall mild on the first day after surgery in cases having a simple multimodal analgesic protocol with their ambulatory breast-conserving and diagnostic procedures.

This is an open access article under the terms of the Creative Commons Attribution License, which permits use, distribution and reproduction in any medium, provided the original work is properly cited.

(c) 2021 The Authors. Acta Anaesthesiologica Scandinavica published by John Wiley \& Sons Ltd on behalf of Acta Anaesthesiologica Scandinavica Foundation 


\section{1 | INTRODUCTION}

Breast cancer $(\mathrm{BC})$ is the most common cancer among women in Norway, and the incidence has increased during the last decades. ${ }^{1}$ Ambulatory BC surgery has been practiced at Oslo University Hospital (OUH), Norway, since 1997 and today approximately 60\% of these patients are discharged the same day. Even though ambulatory surgery for BC is regarded as safe, ${ }^{2,3}$ overnight stay is still predominant in many countries. ${ }^{4-6}$

Only a few hours after surgery, day-surgical patients are discharged home, which means that most of the recovery process takes place out of hospital and professional facilities. Common postdischarge symptoms associated with ambulatory surgery are pain, nau sea and vomiting, ${ }^{7-10}$ which implies discomfort and may prolong the time to mobilization and resumption of normal daily activities. ${ }^{11}$

Postdischarge nausea and vomiting (PDNV) ${ }^{12}$ may cause severe distress as the patient does not have access to fast-onset intravenous (i.v.) anti-emetics at home. The risk for PDNV can be as high as $80 \%$ without prophylaxis ${ }^{8}$ and it may affect more than one-third of the patients even when prophylactic drugs are administered. ${ }^{13,14}$

Previous data from our research group indicated an incidence of $18 \%$ moderate-to-severe pain at rest and $40 \%$ when coughing or stretching the shoulder, during the first $24 \mathrm{~h}$ after $\mathrm{BC}$ surgery. ${ }^{15} \mathrm{At}$ that time, dexamethasone was not a part of the multimodal regimen for pain and nausea prophylaxis.

In this study, we therefore wanted to investigate how ambulatory BC surgery patients experienced the first $24 \mathrm{~h}$ after discharge, especially regarding pain and nausea, after receiving a fixed multimodal prophylactic drug regimen including dexamethasone.

Recently, the Norwegian Research Centre for Health Services published a questionnaire, especially developed for telephone follow-up (TFU) on postoperative day 1 (POD1) after ambulatory surgery. ${ }^{16}$ This facilitated the registration of structured data on postdischarge outcomes.

The primary aims of the study were to describe the incidence and severity of postdischarge pain and PDNV. Secondary aims were to assess if age, type of anaesthesia or surgical procedure were associated with pain or PDNV, and whether there was an association between postdischarge pain and PDNV.

We were also interested in patient-reported satisfaction, adherence to postdischarge analgesic instructions, sleep, resumption of daily activity, wound haemostasis problems and unscheduled health care contacts.

\section{2 | METHODS AND MATERIALS}

The study was a prospective, observational quality assessment study of women who underwent diagnostic or breast-conserving ambulatory surgery for $\mathrm{BC}$. At $\mathrm{OUH}$, the following conditions or classifications are common for BC patients scheduled for ambulatory surgery: fibroadenoma, phyllodes tumour, papilloma, diagnostic biopsies in breast, ductal carcinoma in situ (DCIS) I-III, breast cancer (uni- or bilateral, uni- or multifocal) and sentinel lymph node dissection (SLNB). ASA grade I-III.

As patients with ambulatory mastectomies and axillary lymph node dissection (ALND) were few and also represented a different surgical trauma compared with the rest of the study population, they were excluded from the data analyses (Figure 1).

Patients included in the study received a follow-up call on POD1, and the standardized validated questionnaire was used to collect data. Answering the questionnaire was voluntary, and all data were anonymous. The study protocol was submitted to the Regional Committee for Medical and Health Research Ethics of South-East Norway, and classified as a quality assurance study (ref. nr.2018/1038). The Data Protection Officer at OUH approved the study.

\section{1 | Inclusion criteria}

To be included for TFU on POD1, the patient had to be discharged home on the day of surgery, be 18 years or older and speak Norwegian or English. Patients admitted to overnight stay were excluded.

\subsection{Data collection}

Upon discharge, the patients received a written copy of the questionnaire ${ }^{16}$ (see Appendix) and were informed of the data collection during the phone call next day. The questionnaire included questions on postoperative outcomes such as postdischarge pain, PDNV, sleep, wound hemostasis, resumption of daily activities, adherence to analgesic instructions, unscheduled contact with healthcare services, as well as satisfaction with information, discharge and overall patient satisfaction. Most response alternatives were fixed on a 3-5-point Likert scale, but some 'Yes' or 'No' answers and multiplechoice answers were also present. Patients rated their pain at rest, during activity and 'worst pain since discharge' on a numeric rating scale (NRS) from 0 (no pain at all) to 10 (worst pain imaginable). ${ }^{17,18}$

Assigned nurses at the day surgery unit called the patients on POD1, between 11.00 am and $14.00 \mathrm{pm}$. To make sure the patient felt free to express her opinion, a nurse unknown to the patient performed the call. The data were registered in a day-surgical quality register at $\mathrm{OUH}$. Demographic data on age, surgical procedure and type of anaesthesia were also collected.

\subsection{Anaesthesia and multimodal prophylaxis for pain and nausea}

The standard general anaesthesia procedure was total intravenous anaesthesia (TIVA) with propofol and remifentanil, administered by target-controlled infusions, and ventilation by a laryngeal mask airway using a mixture of oxygen and air. A crystalloid infusion (Ringer-Acetat 500-1000 ml) was administered per- and post-operatively. During the 
FIGURE 1 Breast cancer surgery patients; Flow diagram of screening, enrollment and analysis
Flow Diagram

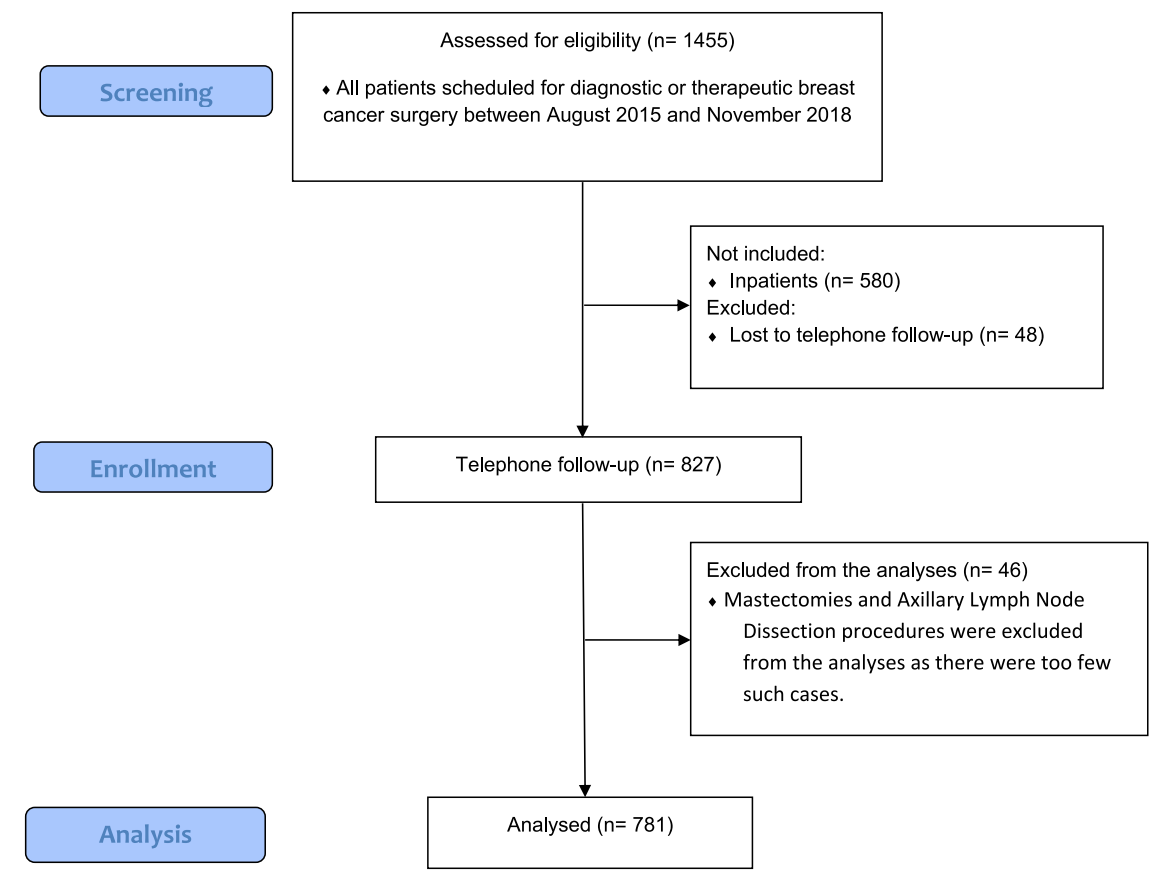

study, there was a time period when it was difficult to receive Propofol from the producer. Therefore volatile anaesthetics was administered to all patients for maintenance. Local anaesthetics combined with sedation was used for some minor diagnostic procedures.

All patients received a standardized multimodal pain- and nausea prophylaxis. For oral pre-medication, the patients had paracetamol $2 \mathrm{~g}$ (1.5 g if weight $<60 \mathrm{~kg}$, or $>60$ years) and a COX-II inhibitor, that is, celecoxib $400 \mathrm{mg}$ ( $200 \mathrm{mg}$ if weight $<60 \mathrm{~kg}$, or $>60$ years), unless contraindicated. Etoricoxib replaced celecoxib in case of sulfonamide-allergy. Peroperatively, the patients received fentanyl i.v. before end of surgery and approximately $20 \mathrm{ml}$ bupivacaine $2.5 \mathrm{mg} / \mathrm{ml}$ as surgical site infiltration for pain prophylaxis. Routine i.v. prophylaxis for postoperative nausea and vomiting was provided with ondansetron $4 \mathrm{mg}$ and dexamethasone $8 \mathrm{mg}$, the latter also being part of analgesic prophylaxis.

Postoperative pain was initially treated with fentanyl i.v. in the post-anaesthesia care unit (PACU). Oral opioids, that is, paracetamo $500 \mathrm{mg} /$ codeine $30 \mathrm{mg}$ or oxycodone $5 \mathrm{mg}$ tablets were administered if needed. The patients had to be free from nausea and adequately pain-relieved, that is, NRS $<4$, to be discharged.

All patients got a prescription for paracetamol and celecoxib to be used prophylactically after discharge. Four tablets of paracetamol $500 \mathrm{mg} /$ codeine $30 \mathrm{mg}$ were routinely given to the patients as rescue analgesia, for use at home. A few patients also received 1-4 tablets of oxycodone $5 \mathrm{mg}$ in addition. All patients received oral and written instructions on analgesics administration.

Nausea or vomiting in the PACU was primarily treated with i.v. metoclopramide $10 \mathrm{mg}$ or a repeated dose of ondansetron. An antihistamine (cyclizine) was added if needed.

\section{4 | Outcome measures}

The 0-10 NRS for pain was further divided into four sections: $0=$ none, $1-3=$ mild, $4-6=$ moderate, $7-10=$ severe $^{17,19,20}$ For statistical analyses in this study, we merged the two latter categories into a '4-10' category of moderate-to-severe pain. ${ }^{19,21}$

Age was divided into three groups: $18-39,40-59$ and 6089 years. Postdischarge nausea, postdischarge vomiting and wound hemostatsis were dichotomized into 'yes' or 'no'. Three different types of anaesthesia were used: TIVA, volatile anaesthetics (i.e. desflurane) and local anaesthetics with sedation. For satisfaction with information, time for discharge and overall patient satisfaction, the response alternatives 'Mostly satisfied/To a large degree' and 'Very satisfied/Yes' were merged into 'yes'.

In consultation with a Senior Consultant at the Department of Breast and Endocrine Surgery (co-author ES), the surgical procedures were classified as described in Table 1.

\section{5 | Statistical analyses}

Continuous data were described with mean and standard deviation (SD) or median and interquartile range (Q1-Q3) when not normally distributed. Categorical data were presented as counts and percentages. Differences between responders and non-responders were analysed using independent-samples $t$ test for continuous data and Pearson's chi-square test for categorical variables. Pearson's chisquare test was also used to assess the possible association between pairs of categorical variables. 
To quantify possible associations between pain (NRS in three categories) and selected variables, we fitted ordinal logistic regression models. The following predictive factors were entered for the three different outcomes of pain (i.e. at rest, during activity, worst pain): age, type of anaesthesia, surgical procedure and nausea. The model assumptions were fulfilled and the model fit was satisfactory.

Associations between a binary outcome of nausea and selected covariates were estimated using multivariate logistic regression with age, type of anaesthesia, surgical procedure and the three different variables of postdischarge pain entered into the model.

Only the surgical categories comprising more than 50 patients were entered into the regression models. The results were expressed as odds ratios (OR) with $95 \%$ confidence intervals $(\mathrm{Cl})$.

All analyses were considered exploratory, so no correction for multiple testing was done and $p$-values $<.05$ were considered statistically significant. IBM SPSS Statistics for Windows, version 25.0 (Armonk, NY: IBM Corp), was used for statistical analyses.

\section{3 | RESULTS}

Between August 2015 and November 2018, a total of 875 patients met the criteria for TFU. Response with TFU on POD1 was successful in 827 (94.5\%), all willing to answer the questionnaire. Patients with mastectomy and ALND $(n=46)$ were excluded from analyses, as described above, leaving 781 patients included for further analyses (Figure 1). Missing rate for each variable in the questionnaire varied between 0 and 2.8\%. The mean age was 51.4 (SD 14.9), ranging

TABLE 1 Patient characteristics

\begin{tabular}{ll}
\hline Age in 3 categories $(n=773)$ & $n(\%)$ \\
$18-39$ & $178(23.0)$ \\
$40-59$ & $334(43.2)$ \\
$60-89$ & $261(33.8)$ \\
Mean (SD) range & $51.4(14.9) 18-85$ \\
Type of anaesthesia $(n=769)$ & $n(\%)$ \\
TIVA & $723(94.0)$ \\
Local anaesthetics + sedation & $21(2.7)$ \\
Volatile anaesthetics & $25(3.3)$ \\
Surgical procedure $(n=781)$ & $n(\%)$ \\
Breast conserving surgery + SLNB & $278(35.6)^{\mathrm{b}}$ \\
Re-excision breast + SLNB & $4(0.5)$ \\
Breast conserving surgery & $58(7.4)^{\mathrm{b}}$ \\
Incisional or diagnostic biopsy from breast & $331(42.4)^{\mathrm{b}}$ \\
SLNB & $12(1.5)$ \\
Re-excision breast & $67(8.6)^{\mathrm{b}}$ \\
Other & $31(4.0)$ \\
\hline
\end{tabular}

Abbreviations: SLNB, sentinel lymph node biopsy; TIVA, total intravenous anaesthesia.

a Other minor operations related to breast cancer surgery.

${ }^{\mathrm{b}}$ Entered into the regression models. from 18 to 85 years. TIVA was used in $94 \%$ of the respondents, and $43 \%$ had breast-conserving surgery (Table 1). Among women with breast-conserving surgery +SLNB, 5.4\% were 18-39 years, and $49.5 \%$ were 60 years or older. Diagnostic biopsies were more common in younger women, $18-39$ years (41.5\%), than in women over 60 years (19.5\%). There was no statistically significant association between type of anaesthesia and surgical procedure, nausea or age.

There were no differences between responders and non-responders concerning the surgical procedure. However, non-responders had almost exclusively TIVA, and were significantly younger than responders, with a mean age of 44.9 (SD 15.2) versus 51.4 (SD 14.9).

\section{1 | Postdischarge pain and adherence to analgesics instructions:}

On POD1, nearly $50 \%$ were totally pain-free (i.e. score of 0 ) at rest, and $94.7 \%$ reported a pain score less than 4 at rest. During the activity, pain was scored less than 4 in $83 \%$ of the patients. When assessing worst pain since discharge, $69.3 \%$ reported a pain score below 4 , whereas $4.3 \%$ reported a worst pain score of 7 or more (Table 2 ).

The adherence to instructions for postdischarge analgesics was high (86.5\%), but $7.6 \%$ reported pain even when following the prescribed doses, and $5.3 \%$ with non-adherence to analgesics instructions reported pain (Table 2). Low pain score was significantly associated with adherence to instructions for analgesics $(p<.001)$.

\subsection{1 | Predictive factors for postdischarge pain}

In the regression analysis, there were no statistically significant differences in pain scores between the four surgical procedures. Patients having local anaesthetics with sedation, were about two time more likely to report postdischarge pain compared to patients with TIVA. This association was statistically borderline significant both at rest and at worst pain (Table 3).

Younger patients (18-59 years) experienced more pain than the oldest (60-89 years); both at rest, during activity and regarding worst pain since discharge (Table 3).

Absence of nausea was significantly associated with lower odds for experiencing high pain scores at rest, during activity and worst pain since discharge $(p<.001)$ (Table 3$)$.

\section{2 | Postdischarge nausea and vomiting}

Nausea occurred in 137 patients (17.7\%), and 9 patients (1.2\%) reported vomiting (Table 4).

There was no association between nausea and age, anaesthetic procedure, type of surgery or pain at rest, but the higher the level of pain during activity, the higher were the odds for nausea. A higher level of worst pain since discharge was also associated with higher odds for nausea (Table 4). 
TABLE 2 Postdischarge pain and adherence to analgesics instructions

\begin{tabular}{ll} 
Postdischarge pain at rest on POD1 $(n=776)$ & $n(\%)$ \\
NRS 0 & $387(49.9)$ \\
NRS 1-3 & $348(44.8)$ \\
NRS 4-6 & $38(4.9)$ \\
NRS 7-10 & $3(0.4)$ \\
Range & $0-8$ \\
Median (Percentiles, Q1-Q3) & $1(0-2)$ \\
\hline Postdischarge pain during activity on POD1 $(n=772)$ & $\mathrm{n}(\%)$ \\
NRS 0 & $232(30.1)$ \\
NRS 1-3 & $409(52.9)$ \\
NRS 4-6 & $122(15.8)$ \\
NRS 7-10 & $9(1.2)$ \\
Range & $0-9$ \\
Median (Percentiles, Q1-Q3) & $1(0-3)$ \\
Worst postdischarge pain since discharge $(n=774)$ & $\mathrm{n}(\%)$ \\
NRS 0 & $171(22.1)$ \\
NRS 1-3 & $366(47.2)$ \\
NRS 4-6 & $204(26.4)$ \\
NRS 7-10 & $33(4.3)$ \\
Range & $0-9$ \\
Median (Percentiles, Q1-Q3) & \\
I have not followed the recommendations and I am & $41(5.3)$ \\
analgesics instructions ( $=773)$ & \\
I do not need any pain medication & $\mathrm{n}(\%)$ \\
I have taken pain medication as recommended and & $610(78.9)$ \\
\hline
\end{tabular}

Abbreviations: NRS, Numeric rating scale; POD1, Postoperative day 1; Q1-Q3, Interquartile range.

\section{3 | Patient satisfaction and other postdischarge data}

During the first night after surgery, 64.8\% slept less than normal, stating the most common causes were unusual sleeping position (26\%), restlessness (16.4\%) and pain (9.1\%). On POD1, 81.9\% were back to normal or close to normal daily activity. Twenty-five patients (3.2\%) made unanticipated contact with healthcare services after discharge. No patient had serious complications. The overall patient satisfaction of the day-surgical concept was $99.2 \%$, and satisfaction with information and time for discharge were also high (Table 5).

\section{4 | DISCUSSION}

The incidence of $24 \mathrm{~h}$ postdischarge pain and nausea after diagnostic and breast-conserving ambulatory BC surgery was low in the
TABLE 3 Ordinal logistic regression for postdischarge pain

\begin{tabular}{|c|c|c|c|}
\hline Variable & OR & $95 \% \mathrm{Cl}$ & $p$-value \\
\hline \multicolumn{4}{|c|}{ A) Postdischarge pain at rest POD1 } \\
\hline \multicolumn{4}{|l|}{ Age } \\
\hline $18-39$ & 3.98 & $2.48 ; 6.37$ & $<.001$ \\
\hline $40-59$ & 2.12 & $1.49 ; 3.02$ & $<.001$ \\
\hline $60-89$ (ref.) & 1 & & \\
\hline \multicolumn{4}{|l|}{ Nausea } \\
\hline No nausea & 0.42 & $0.28 ; 0.63$ & $<.001$ \\
\hline Nausea (ref.) & 1 & & \\
\hline \multicolumn{4}{|l|}{ Type of anaesthesia } \\
\hline Volatile anaesthetics & 2.10 & $0.91 ; 4.84$ & .08 \\
\hline $\begin{array}{l}\text { Local anaesthetics + } \\
\text { sedation }\end{array}$ & 2.60 & $1.00 ; 6.79$ & .05 \\
\hline TIVA (ref.) & 1 & & \\
\hline \multicolumn{4}{|l|}{ Surgical procedure } \\
\hline $\begin{array}{l}\text { Breast conserving } \\
\text { surgery + SLNB }\end{array}$ & 1.02 & $0.58 ; 1.79$ & .96 \\
\hline $\begin{array}{l}\text { Breast conserving } \\
\text { surgery }\end{array}$ & 1.11 & $0.52 ; 2.33$ & .79 \\
\hline $\begin{array}{l}\text { Incision or diagnostic } \\
\text { biopsy breast }\end{array}$ & 0.76 & $0.43 ; 1.36$ & .35 \\
\hline Re-excision breast (ref.) & 1 & & \\
\hline
\end{tabular}

B) Postdischarge pain during activity POD1

Age

18-39

5.57

3.47; $8.95<.001$

40-59

2.38

$1.70 ; 3.34<.001$

$60-89$ (ref.)

1

Nausea

No nausea

0.33

$0.22 ; 0.49<.001$

Nausea (ref.)

1

Type of anaesthesia

\begin{tabular}{|c|c|c|}
\hline Volatile anaesthetics & 1.02 & $0.45 ; 2.31$ \\
\hline $\begin{array}{l}\text { Local anaesthetics + } \\
\text { sedation }\end{array}$ & 2.26 & $0.89 ; 5.74$ \\
\hline
\end{tabular}

Surgical procedure

$\begin{array}{llll}\begin{array}{l}\text { Breast conserving } \\ \quad \text { surgery + SLNB }\end{array} & 1.30 & 0.75 ; 2.23 & .35 \\ \begin{array}{l}\text { Breast conserving } \\ \quad \text { surgery }\end{array} & 1.48 & 0.72 ; 3.05 & .28 \\ \begin{array}{l}\text { Incision or diagnostic } \\ \quad \text { biopsy breast }\end{array} & 0.90 & 0.52 ; 1.58 & .73 \\ \begin{array}{l}\text { Re-excision breast (ref.) } \\ \text { Re- }\end{array} & 1 & & \end{array}$

C) Worst pain since discharge

Age

$\begin{array}{llll}18-39 & 5.45 & 3.43 ; 8.64 & <.001 \\ 40-59 & 2.30 & 1.65 ; 3.21 & <.001 \\ 60-89 \text { (ref.) } & 1 & & \end{array}$

(Continues) 
TABLE 3 (Continued)

\begin{tabular}{|c|c|c|c|}
\hline Variable & OR & $95 \% \mathrm{Cl}$ & $p$-value \\
\hline \multicolumn{4}{|l|}{ Nausea } \\
\hline No nausea & 0.31 & $0.21 ; 0.46$ & $<.001$ \\
\hline Nausea (ref.) & 1 & & \\
\hline \multicolumn{4}{|l|}{ Type of anaesthesia } \\
\hline Volatile anaesthetics & 1.27 & $0.56 ; 2.86$ & .57 \\
\hline $\begin{array}{l}\text { Local anaesthetics + } \\
\text { sedation }\end{array}$ & 2.60 & $1.01 ; 6.73$ & .05 \\
\hline TIVA (ref.) & 1 & & \\
\hline \multicolumn{4}{|l|}{ Surgical procedure } \\
\hline $\begin{array}{l}\text { Breast conserving } \\
\text { surgery + SLNB }\end{array}$ & 0.96 & $0.56 ; 1.63$ & .87 \\
\hline $\begin{array}{l}\text { Breast conserving } \\
\text { surgery }\end{array}$ & 0.97 & $0.48 ; 1.96$ & .93 \\
\hline $\begin{array}{l}\text { Incision or diagnostic } \\
\text { biopsy breast }\end{array}$ & 0.67 & $0.39 ; 1.16$ & .15 \\
\hline Re-excision breast (ref.) & 1 & & \\
\hline
\end{tabular}

Abbreviations: POD1, postoperative day 1; SLNB, sentinel lymph node biopsy; TIVA, total intravenous anaesthesia.

present study, using a simple, multimodal prophylaxis regimen and proper instructions for use. There was a strong association between higher pain scores and younger age and also between higher pain scores and nausea.

\section{1 | Postdischarge pain}

Pain intensity is usually at its highest on the day after surgery ${ }^{22}$ but only $5.3 \%$ of our patients reported moderate-to-severe pain at rest on POD1. In a similar study population, Ballardini et al. ${ }^{5}$ found an incidence of $6.7 \%$ moderate-to-severe pain, also with the use of multimodal analgesic techniques. In contrast, Susini et al. ${ }^{6}$ recently reported $40.6 \%$ postdischarge pain of NRS 4 or higher after ambulatory BC surgical procedures comparable to ours, but with a less extensive pain prophylaxis. In our study, younger patients had significantly 2-5 times higher odds for postdischarge pain than older patients, which is similar to prior studies. ${ }^{20,22-25}$

We found no association between pain and surgical procedure, which is in contrast to the study by Rehberg et al. ${ }^{24}$ Our result may be related to the overall low surgical invasiveness in our day-surgical study population. At $\mathrm{OUH}$, all patients with planned mastectomy are offered an immediate reconstructive procedure, unless contraindicated, and subsequently transferred to inpatient care. Patients with axillary lymph node dissection (ALND) and extensive oncoplastic operations are usually admitted for an overnight stay because of surgical drains.

Type of anaesthesia may affect both nausea and pain after surgery. Propofol-based TIVA seems to be associated with a lower risk for postoperative nausea and less pain during the first $12 \mathrm{~h}$ after surgery. ${ }^{26,27}$ Whether type of anaesthesia affects pain-perception as long as $24 \mathrm{~h}$ after surgery is uncertain, but propofol-based anaesthesia has been reported to reduce the need for analgesics up to
24 h postoperatively. ${ }^{26}$ Patients receiving volatile anaesthetics and especially local anaesthetics with sedation in our study, reported more pain at rest and also a higher worst-pain score, than patients with TIVA. However, there were few patients treated with local anaesthetics with sedation or volatile anaesthetics, and therefore we do not have sufficient statistical power to conclude on the effects of these anaesthetic methods on postoperative pain.

Recent pain-related research on BC surgery focuses on the benefits of invasive blocks, such as the paravertebral (PVB) or pectoral nerves blocks (PECS I or II), although these may not be needed for routine day cases. ${ }^{28}$ Our results may indicate that the multimodal prophylaxis regimen used in the study, with no nerve blocks, is adequate for ordinary ambulatory $\mathrm{BC}$ surgical procedures. The regimen is also in accordance with the PROSPECT pain management recommendations for oncological breast surgery, ${ }^{29}$ except for the non-use of gabapentinoids in our setting. Gabapentinoids are known for sedation-related adverse effects, ${ }^{30}$ and should be used with caution in ambulatory surgery, due to reports on dizziness and patients falling after coming home. ${ }^{31}$

The high adherence to detailed advice on postdischarge analgesics in our study may explain the rather low incidence of moderate-to-severe pain. However, for $7.6 \%$ of the patients the prescribed drug regimen was proved less than adequate. This is a problem also reported by Fahmy et al., ${ }^{32}$ who experienced that $33 \%$ rated the efficacy of discharge analgesics as inadequate. Non-adherence to analgesics instructions is another common problem ${ }^{23,33,34}$ that may lead to unnecessary pain. In our study, $5.3 \%$ were non-adherent and still had pain, that is, prophylactic analgesics were not used as prescribed, or they had not taken the opioids they had received as rescue medication. Recently, Valeberg et al. ${ }^{23}$ reported $37 \%$ non-adherence to instructions on analgesic use, even though an intervention with extensive information and follow-up was applied. A frequent cause for the non-adherence in that study was that patients rather endured pain than risking the common known side-effects of the opioids. ${ }^{23}$

\section{2 | Postdischarge nausea and vomiting}

Female gender is an independent risk factor for PDNV, ${ }^{8}$ which is relevant since all participants in our study were women. Even though all patients received two standard prophylactic anti-emetics, and only $3.3 \%$ received volatile anaesthesia, $17.8 \%$ experienced nausea and $1.2 \%$ vomited after returning home. In contrast, Ballardini et al. ${ }^{5}$ reported only $1.5 \%$ nausea, but among the patients converted to inpatients in their study, $22 \%$ were admitted because of persistent nausea/vomiting. Wesmiller et al. ${ }^{14}$ on the other hand, recently reported $35 \%$ nausea and $8.2 \%$ vomiting up to $48 \mathrm{~h}$ after BC surgery, when using a multimodal prophylaxis. They found that pain scores and quantity of opioids taken postoperatively were significantly higher for women with PDNV, which is in consistent with previous research. ${ }^{8,35}$ In our study, we have no exact data on postdischarge opioid-consumption, but the patients had the option of using 
TABLE 4 Postdischarge nausea and vomiting

\begin{tabular}{|c|c|c|c|}
\hline \multicolumn{3}{|l|}{ Postdischarge nausea $(n=772)$} & $\mathrm{n}(\%)$ \\
\hline \multicolumn{3}{|l|}{ Not at all } & $635(82.3)$ \\
\hline \multicolumn{3}{|l|}{ To some degree } & $127(16.4)$ \\
\hline \multicolumn{3}{|l|}{ To a large degree } & $10(1.3)$ \\
\hline \multicolumn{4}{|l|}{ Postdischarge vomiting $(n=759)$} \\
\hline Not at all & & \multicolumn{2}{|c|}{$750(98.8)$} \\
\hline To some degree & & \multicolumn{2}{|c|}{$7(0.9)$} \\
\hline To a large degree & & \multicolumn{2}{|c|}{$2(0.3)$} \\
\hline \multicolumn{4}{|c|}{ Multivariate logistic regression for postdischarge nausea } \\
\hline Variable & OR & $95 \% \mathrm{Cl}$ & $p$-value \\
\hline Age & 0.99 & $0.98 ; 1.01$ & .46 \\
\hline \multicolumn{4}{|l|}{ Type of anaesthesia } \\
\hline TIVA & 0.76 & $0.27 ; 2.18$ & .61 \\
\hline Local anaesthetics + sedation & 0.69 & $0.14 ; 3.43$ & .65 \\
\hline Volatile anaesthetics (ref) & 1 & & \\
\hline \multicolumn{4}{|l|}{ Surgical procedure } \\
\hline Re-excision breast & 0.84 & $0.37 ; 1.89$ & .67 \\
\hline Incision/Diagnostic biopsy breast & 0.94 & $0.56 ; 1.57$ & .82 \\
\hline Breast conserving surgery & 0.65 & $0.26 ; 1.59$ & .34 \\
\hline Breast conserving surgery + SLNB (ref.) & 1 & & \\
\hline Pain at rest & 0.98 & $0.79 ; 1.22$ & .86 \\
\hline Pain during activity & 1.29 & $1.04 ; 1.61$ & .02 \\
\hline Worst pain since discharge & 1.19 & $1.00 ; 1.40$ & .05 \\
\hline
\end{tabular}

Abbreviations: SLNB, sentinel lymph node biopsy; TIVA, total intravenous anaesthesia. codeine as a part of their postdischarge analgesics regimen, and were instructed to use them if pain was medium or strong. A few patients had access to oxycodone in addition. As most of our patients adhered to the instructions, we may assume that a high number of our patients used opioids after discharge. This may partly explain the strong association between pain and nausea in our study, that is, pain initiates the use of opioids with subsequent nausea. However, we cannot rule out an alternative hypothesis that pain per se may provoke nausea. This may be an area of further studies with better monitoring of timing sequence between onset of pain and nausea, and subsequent results of rescue treatment for either.

\section{3 | Patient satisfaction}

There is no consensus on how to define patient satisfaction, ${ }^{36,37}$ even though satisfaction is commonly used to document quality in health care. Jaensson et al. ${ }^{37}$ suggest that both patients' experiences and expectations of care will influence the overall level of satisfaction. A systematic review of ambulatory BC surgery reported high patient satisfaction, ${ }^{2}$ similar to our results. The patients in our study expressed high satisfaction with information, time for discharge and overall satisfaction. The satisfaction with information provided by the nurse was somewhat higher than by the surgeon, which is in consistence with the study by Marchal et al. ${ }^{38}$

\subsection{Strengths and limitations}

The study was performed at a single center, which may question the generalizability of the results. However, a large sample size with a high response rate, standardized pain- and nausea prophylaxis and perioperative routines, may enhance the validity of our findings.

The non-registration of exact opioid consumption after discharge is a limitation in the interpretation of incidence of pain and nausea, and also the pain-nausea relationship.

The risk for recall bias should be limited as data were collected on POD1. Trained nurses in dialogue with the patient during data collection may have contributed to the quality of the data. The questionnaire we used was especially developed for TFU; therefore, a slight limitation lies within the restricted amount of data, even though all seems clinically relevant.

Despite the large sample size, there was limited precision in some of our estimates as reflected in broad confidence intervals. Thus an even larger sample would provide a higher level of statistical power.

\section{5 | CONCLUSION}

Postdischarge pain scores and nausea-incidence were generally low on the day after surgery, when using a simple multimodal oral- and i.v. pain- and nausea prophylaxis. Young age was a strong predictor for postdischarge 
(C) (C) Scandinavica

TABLE 5 Other postdischarge assessments and patient satisfaction

\begin{tabular}{|c|c|}
\hline How did you sleep the first night after surgery $(n=778)$ & $\mathrm{n}(\%)$ \\
\hline Not at all & 30 (3.9) \\
\hline To some degree & $272(34.9)$ \\
\hline To a large degree & $202(26.0)$ \\
\hline Normal sleep & $274(35.2)$ \\
\hline Cause of impaired sleep ${ }^{a}(n=504)$ & $\mathrm{n}(\%)^{\mathrm{b}}$ \\
\hline Pain & $68(9.1)$ \\
\hline Restlessness & $123(16.4)$ \\
\hline Headache & $30(4.0)$ \\
\hline Nightmare & $6(0.8)$ \\
\hline Unusual sleeping position & $195(26.0)$ \\
\hline Other & $197(26.2)$ \\
\hline Resumption of normal daily activity POD1 $(n=766)$ & $n(\%)$ \\
\hline Not at all & $9(1.2)$ \\
\hline To some degree & $130(17.0)$ \\
\hline To a large degree & $248(32.4)$ \\
\hline Normal activity & $379(49.5)$ \\
\hline $\begin{array}{l}\text { Bleeding from surgical wound after discharge } \\
\qquad(n=762)\end{array}$ & $\mathrm{n}(\%)$ \\
\hline Not at all & $748(98.2)$ \\
\hline To some degree & $14(1.8)$ \\
\hline To a large degree & 0 \\
\hline $\begin{array}{l}\text { Contact with health care services after discharge } \\
\qquad(n=776)\end{array}$ & $n(\%)$ \\
\hline No & $751(96.8)$ \\
\hline Yes & $25(3.2)$ \\
\hline $\begin{array}{l}\text { Cause of contact with health services after discharge }{ }^{a} \\
\qquad(n=26)\end{array}$ & $n(\%)^{b}$ \\
\hline Pain & $2(0.3)$ \\
\hline Bleeding & $4(0.5)$ \\
\hline Nausea/Vomiting & 0 \\
\hline Breathing problems & 0 \\
\hline Heart palpitations & 0 \\
\hline Fainting & 0 \\
\hline Other & $20(2.6)$ \\
\hline If yes: Who did you contact? $(n=25)$ & $n(\%)^{b}$ \\
\hline Day surgery unit & $7(0.9)$ \\
\hline Emergency department & $6(0.8)$ \\
\hline Outpatient clinic & $11(1.4)$ \\
\hline Surgeon & 0 \\
\hline Emergency Medical Service & 0 \\
\hline General Practitioner & $1(0.1)$ \\
\hline Other & 0 \\
\hline Satisfaction with information given by the nurse $(n=770)$ & $n(\%)$ \\
\hline Not at all & $1(0.1)$ \\
\hline Somewhat satisfied & $1(0.1)$ \\
\hline Mostly satisfied & $87(11.3)$ \\
\hline Very satisfied & $681(88.4)$ \\
\hline
\end{tabular}

TABLE 5 (Continued)

\begin{tabular}{ll}
$\begin{array}{l}\text { Satisfaction with information given by the doctor/ } \\
\text { surgeon }(n=774)\end{array}$ & $\mathrm{n}(\%)$ \\
Not at all & $4(0.5)$ \\
Somewhat satisfied & $13(1.7)$ \\
Mostly satisfied & $124(16.0)$ \\
Very satisfied & $633(81.8)$ \\
\hline Satisfaction with time for discharge $(n=772)$ & $\mathrm{n}(\%)$ \\
Not at all & 0 \\
To some degree & $9(1.2)$ \\
To a large degree & $55(7.1)$ \\
Yes & $708(91.7)$ \\
Overall patient satisfaction $(n=778)$ & $\mathrm{n}(\%)$ \\
Not at all & $2(0.3)$ \\
Somewhat satisfied & $4(0.5)$ \\
Mostly satisfied & $74(9.5)$ \\
Very satisfied & $698(89.7)$
\end{tabular}

Abbreviation: POD1, Postoperative day 1.

${ }^{\text {a } M o r e ~ t h a n ~ o n e ~ a n s w e r ~ w a s ~ p o s s i b l e . ~}$

${ }^{b}$ Valid percent of the study population.

pain in this study. A higher level of worst pain and high pain during activity were associated with increased risk for nausea. There were no serious complications, few unscheduled re-contacts and patient satisfaction was high.

\section{ACKNOWLEDGEMENTS}

We would like to thank the nurses at the PACU/day surgery unit at Oslo University Hospital for contributing to the data-collection.

\section{CONFLICT OF INTEREST}

The authors have no conflicts of interest.

\section{AUTHOR CONTRIBUTIONS}

Study design: Mi Stjernberg, Johan C. Raeder, and Tone Rustoen; Data collection: Mi Stjernberg; Analysis; Mi Stjernberg, Milada C. Småstuen, Johan C. Raeder, and Ellen Schlichting; and Manuscript preparation: Mi Stjernberg, Johan C. Raeder, Tone Rustoen, Berit T. Valeberg, Ellen Schlichting, and Milada C. Småstuen.

\section{ORCID}

Mi Stjernberg (D) https://orcid.org/0000-0002-2114-4965

\section{REFERENCES}

1. Kreftregisteret. Årsrapport 2019 med resultater og forbedringstiltak fra Nasjonalt kvalitetsregister for brystkreft. Oslo 2020 [cited 2021 22.06]. https://www.kreftregisteret.no/Generelt/Rapporter/ Arsrapport-fra-kvalitetsregistrene/Arsrapport-for-brystkreft/arsra pport-for-brystkreft-2019/

2. Marla S, Stallard S. Systematic review of day surgery for breast cancer. Int J Surg. 2009;7(4):318-323.

3. Cordeiro E, Jackson T, Cil T. Same-day major breast cancer surgery is safe: an analysis of short-term outcomes using NSQIP data. Ann Surg Oncol. 2016;23(8):2480-2486. 
4. Rovera F, Ferrari A, Marelli M, et al. Breast cancer surgery in an ambulatory setting. Int J Surg. 2008;6:S116-S118.

5. Ballardini B, Cavalli M, Manfredi GF, et al. Surgical treatment of breast lesions at a day centre: experience of the european institute of oncology. The Breast. 2016;27:169-174.

6. Susini T, Carriero C, Tani F, et al. Day surgery management of early breast cancer: feasibility and psychological outcomes. Anticancer Res. 2019;39(6):3141-3146.

7. Brattwall $M$, Warren Stomberg $M$, Rawal $N$, Segerdahl $M$, Jakobsson J, Houltz E. Patients' assessment of 4-week recovery after ambulatory surgery. Acta Anaesthesiol Scand. 2011;55(1):9298. doi:10.1111/j.1399-6576.2010.02322.x

8. Apfel C, Philip B, Cakmakkaya O, et al. Who is at risk for postdischarge nausea and vomiting after ambulatory surgery? Anesthesiology. 2012;117(3):475-486.

9. Odom-Forren J, Rayens MK, Gokun Y, et al. The relationship of pain and nausea in postoperative patients for 1 week after ambulatory surgery. Clin J Pain. 2015;31(10):845-851.

10. Wallden J, Flodin J, Hultin M. Validation of a prediction model for post-discharge nausea and vomiting after general anaesthesia in a cohort of Swedish ambulatory surgery patients. Eur J Anaesthesiol. 2016;33(10):743-749.

11. Rosén HI, Bergh IH, Odén A, Mårtensson LB. Patients' experiences of pain following day surgery - at 48 hours, seven days and three months. Open Nurs J. 2011;5:52-59.

12. ASPAN'S evidence-based clinical practice guideline for the prevention and/or management of PONV/PDNV. J Perianesth Nurs. 2006;21(4):230-250.

13. Odom-Forren J. Measurement of postdischarge nausea and vomiting for ambulatory surgery patients: a critical review and analysis. $J$ Perianesth Nurs. 2011;26(6):372-383.

14. Wesmiller SW, Bender CM, Conley YP, et al. A prospective study of nausea and vomiting after breast cancer surgery. $J$ Perianesth Nurs. 2017;32(3):169-176.

15. Hval K, Thagaard KS, Schlichting E, Raeder J. The prolonged postoperative analgesic effect when dexamethasone is added to a nonsteroidal antiinflammatory drug (rofecoxib) before breast surgery. Anesth Analg. 2007;105(2):481-486.

16. Nasjonal fagprosedyre. Dagkirurgiske pasienter: oppfølgingssamtale via telefon første postoperative dag: Helsebiblioteket.no; [cited 2021 23.06]. https://www.helsebiblioteket.no/fagprosedy rer/ferdige/dagkirurgiske-pasienter-oppfolgingssamtale-meddagkirurgiske-pasienter-via-telefon-forste-postoperative-dag

17. Breivik $\mathrm{H}$, Borchgrevink PC, Allen SM, et al. Assessment of pain. $\mathrm{Br}$ J Anaesth. 2008;101(1):17-24.

18. Hjermstad MJ, Fayers PM, Haugen DF, et al. Studies comparing numerical rating scales, verbal rating scales, and visual analogue scales for assessment of pain intensity in adults: a systematic literature review. J Pain Symptom Manage. 2011;41(6):1073-1093.

19. Gerbershagen HJ, Rothaug J, Kalkman CJ, Meissner W. Determination of moderate-to-severe postoperative pain on the numeric rating scale: a cut-off point analysis applying four different methods. Br J Anaesth. 2011;107(4):619-626.

20. Gerbershagen HJ, Pogatzki-Zahn E, Aduckathil S, et al. Procedurespecific risk factor analysis for the development of severe postoperative pain. Anesthesiology. 2014;120(5):1237-1245.

21. Beauregard L, Pomp A, Choinière M. Severity and impact of pain after day-surgery. Can J Anaesth. 1998;45(4):304.

22. Schnabel A, Yahiaoui-Doktor M, Meissner W, Zahn PK, PogatzkiZahn EM. Predicting poor postoperative acute pain outcome in adults: an international, multicentre database analysis of risk factors in 50,005 patients. Pain Reports. 2020;5(4):e831.

23. Valeberg BT, Dihle A, Småstuen MC, Endresen AO; Rustøen TJJoCN. The effects of a psycho-educational intervention to improve pain management after day surgery: a randomized clinical trial. J Clin Nurs. 2021;30:1132-1143.
24. Rehberg B, Mathivon S, Combescure C, Mercier Y, Savoldelli GL. Prediction of acute postoperative pain following breast cancer surgery using the pain sensitivity questionnaire: a cohort study. Clin J Pain. 2017;33(1):57-66.

25. Habib AS, Kertai MD, Cooter M, Greenup RA, Hwang S. Risk factors for severe acute pain and persistent pain after surgery for breast cancer: a prospective observational study. Reg Anesth Pain Med. 2019;44(2):192-199.

26. Peng K, Liu H-Y, Wu S-R, et al. Does propofol anesthesia lead to less postoperative pain compared with inhalational anesthesia?: a systematic review and meta-analysis. Anesth Analg. 2016;123(4):846-858.

27. Schraag S, Pradelli L, Alsaleh AJO, et al. Propofol vs. inhalational agents to maintain general anaesthesia in ambulatory and in-patient surgery: a systematic review and meta-analysis. BMC Anesthesiol. 2018;18(1):162.

28. Barrington MJ, Seah GJ, Gotmaker R, Lim D, Byrne K. Quality of recovery after breast surgery: a multicenter randomized clinical trial comparing pectoral nerves interfascial plane (Pectoral Nerves II) block with surgical infiltration. Anesth Analg. 2020;130(6):1559-1567.

29. Jacobs A, Lemoine A, Joshi GP, et al. PROSPECT guideline for oncological breast surgery: a systematic review and procedure-specific postoperative pain management recommendations. Anaesthesia. 2020;75(5):664-673.

30. Barazanchi A, MacFater WS, Rahiri J-L, et al. Evidence-based management of pain after laparoscopic cholecystectomy: a PROSPECT review update. Br J Anaesth. 2018;121(4):787-803.

31. Raeder J. Procedure-specific and patient-specific pain management for ambulatory surgery with emphasis on the opioid crisis. Curr Opin Anesthesiol. 2020;33:1-7.

32. Fahmy N, Siah J, Umo-Etuk J. Patient compliance with postoperative analgesia after day case surgery: a multisite observational study of patients in North East London. Br J Pain. 2016;10(2):84-89.

33. Stessel B, Theunissen M, Marcus MA, et al. Prevalence and predictors of patient nonadherence to pharmacological acute pain therapy at home after day surgery: a prospective cohort study. Pain Practice. 2018;18(2):194-204.

34. Callebaut I, Jorissen S, Pelckmans C, et al. Four-week pain profile and patient non-adherence to pharmacological pain therapy after day surgery. Anesth Pain Med. 2020;10(3).

35. Apfel CC, Heidrich FM, Jukar-Rao S, et al. Evidence-based analysis of risk factors for postoperative nausea and vomiting. $\mathrm{Br} J$ Anaesth. 2012;109(5):742-753.

36. Al-Abri R, Al-Balushi A. Patient satisfaction survey as a tool towards quality improvement. Oman Med J. 2014;29(1):3-7.

37. Jaensson M, Dahlberg K, Nilsson U. Factors influencing day surgery patients' quality of postoperative recovery and satisfaction with recovery: a narrative review. Perioper Med. 2019;8(1):3.

38. Marchal F, Dravet F, Classe JM, et al. Post-operative care and patient satisfaction after ambulatory surgery for breast cancer patients. Eur J Surg Oncol. 2005;31(5):495-499.

\section{SUPPORTING INFORMATION}

Additional supporting information may be found in the online version of the article at the publisher's website.

How to cite this article: Stjernberg M, Schlichting E, Rustoen T, Valeberg BT, Småstuen MC, Raeder JC. Postdischarge pain, nausea and patient satisfaction after diagnostic and breast-conserving ambulatory surgery for breast cancer: $A$ cross-sectional study. Acta Anaesthesiol Scand. 2021;00:1-9. doi:10.1111/aas.14015 